\title{
Perimeter Approximation of Convex Discs in the Hyperbolic Plane and on the Sphere
}

\author{
Ferenc Fodor ${ }^{1}$ \\ Received: 29 October 2019 / Revised: 1 November 2020 / Accepted: 15 February 2021 / \\ Published online: 8 April 2021 \\ (C) The Author(s) 2021
}

\begin{abstract}
Eggleston (Approximation to plane convex curves. I. Dowker-type theorems. Proc. Lond. Math. Soc. 7, 351-377 (1957)) proved that in the Euclidean plane the best approximating convex $n$-gon to a convex disc $K$ is always inscribed in $K$ if we measure the distance by perimeter deviation. We prove that the analogue of Eggleston's statement holds in the hyperbolic plane, and we give an example showing that it fails on the sphere.
\end{abstract}

Keywords Convex discs in the hyperbolic plane and sphere - Approximation with respect to perimeter deviation - Dowker type theorems

Mathematics Subject Classification 52A55 · 52A27 · 52A40

\section{Introduction and Main Results}

We call a compact, convex set $K \subset \mathbb{R}^{2}$ whose interior is non-empty a convex disc. The perimeter of $K$ is denoted by Per $K$. Let $K$ and $L$ be both convex discs. The perimeter deviation of $K$ and $L$ is defined as

$$
\operatorname{dev}(K, L)=\operatorname{Per}(K \cup L)-\operatorname{Per}(K \cap L) .
$$

We note that although the perimeter deviation is often used to measure the distance of convex figures, it does not define a proper metric on the set of all convex discs as it does not satisfy the triangle inequality, see Besau et al. [2, Appendix A]. However, perimeter deviation is an important concept as it is an example of intrinsic volume

\section{Editor in Charge: János Pach}

Ferenc Fodor

fodorf@math.u-szeged.hu

1 Department of Geometry, Bolyai Institute, University of Szeged, Aradi vértanúk tere 1, Szeged 6720, Hungary 
deviations, which are used to measure distance in approximations of convex bodies by polytopes, see [2, p. 2, (2)]. For another notion of perimeter deviation, which is in fact a metric, see, for example, Florian [9] and references therein.

Eggleston [5], among other questions, investigated how well a convex disc can be approximated by convex polygons of a given number of vertices in the sense of perimeter deviation. For a positive integer $n \geq 3$, let $\mathcal{P}(n)$ denote the set of convex polygons with at most $n$ vertices. Let

$$
\delta_{\mathrm{dev}}(K, n)=\inf \{\operatorname{dev}(K, P): P \in \mathcal{P}(n)\} .
$$

A simple compactness argument shows that for each convex disc $K$ and positive integer $n \geq 3$, there exists a $P \in \mathcal{P}(n)$ which minimises the perimeter deviation from $K$, that is, $\operatorname{dev}(K, P)=\delta_{\operatorname{dev}}(K, n)$. Eggleston proved the following beautiful statement, cf. [5, p. 353, Lemma 4].

Theorem 1.1 Let $K$ be a convex disc and $n \geq 3$ a positive integer. If $P \in \mathcal{P}(n)$ is such that $\operatorname{dev}(K, P)=\delta_{\operatorname{dev}}(K, n)$, then $P$ is inscribed in $K$, that is, $P \subset K$ and the vertices of $P$ are on the boundary of $K$.

According to a classical result of Dowker [4], the minimum area of convex $n$-gons containing a given convex disc $K$ is a convex function of $n$, and the maximum area of convex $n$-gons contained in $K$ is a concave function of $n$. This result was later extended for perimeter in place of area by László Fejes Tóth [7], Eggleston [5], and Molnár [12], independently from each other. Thus, it follows from Theorem 1.1 that for a fixed convex disc $K$, the minimum perimeter deviation of convex $n$-gons from $K$ is also a concave function of $n$.

Let $\mathbb{H}^{2}$ denote the hyperbolic plane, and for two points $p, q \in \mathbb{H}^{2}$ let $d_{H}(p, q)$ denote their hyperbolic distance, and $p q$ denote the segment with endpoints $p$ and $q$. Let $\mathcal{P}_{H}(n)$ be the set of all convex polygons in $\mathbb{H}^{2}$ with at most $n$ vertices for $n \geq 3$. Similarly to the Euclidean case, we define

$$
\delta_{\operatorname{dev}_{H}}(K, n)=\inf \left\{\operatorname{dev}(K, P): P \in \mathcal{P}_{H}(n)\right\} .
$$

For any fixed $K$ and positive integer $n \geq 3$, there exists a convex polygon $P \in \mathcal{P}_{H}(n)$ such that $\operatorname{dev}_{H}(K, P)=\delta_{\operatorname{dev}_{H}}(K, n)$. We extend Theorem 1.1 to the hyperbolic plane $\mathbb{H}^{2}$ as follows.

Theorem 1.2 Let $K$ be a convex disc in $\mathbb{H}^{2}$ and $n \geq 3$ a positive integer. If $P \in \mathcal{P}_{H}(n)$ is such that $\operatorname{dev}(K, P)=\delta_{\operatorname{dev}_{H}}(K, n)$, then $P$ is inscribed in $K$, that is, $P \subset K$ and the vertices of $P$ are on the boundary of $K$.

The analogues of Dowker's theorem both for area and perimeter also hold on the sphere $\mathbb{S}^{2}$ and the hyperbolic plane $\mathbb{H}^{2}$. These were proved by Molnár [12] and László Fejes Tóth [8]. Thus, Theorem 1.2, combined with the hyperbolic version of Dowker's theorem for the maximum perimeter of convex (hyperbolic) $n$-gons contained in a given convex disc $K$, implies the following statement. 
Corollary 1.3 The minimum perimeter deviation of convex $n$-gons from a given convex disc $K$ is a concave function of $n$ in the hyperbolic plane $\mathbb{H}^{2}$.

On the unit sphere $\mathbb{S}^{2}$, the distance of two non-antipodal points $p, q$ is the length of the shorter arc of the unique great circle through $p$ and $q$. The distance of two antipodal points is $\pi$. We call a closed set $K$ on $\mathbb{S}^{2}$ a (spherically) convex disc if it is contained in an open hemisphere and, for any $p, q \in K$, the shorter arc of the unique great circle connecting $p$ and $q$ is also contained in $K$. One may naturally define the perimeter deviation $\operatorname{dev}_{S}(K, L)$ of two convex discs $K, L$ on the unit sphere as in the Euclidean plane and hyperbolic plane. Again, for a convex disc $K$ and $n \geq 3$, there exists a convex spherical polygon $P$ with at most $n$ vertices such that $\operatorname{dev}_{S}(K, P)=\delta_{\operatorname{dev}_{S}}(K, n)$. However, $P$ may not necessarily be contained in $K$ (or contain $K$ ) as shown by the example in Sect. 3.

We note that approximations of convex bodies in $d$-dimensional Euclidean space with respect to all intrinsic volume deviations, where the relative position of the polytope and body is not restricted, have recently been studied by Besau et al. [2]. They prove asymptotic estimates for best approximations of the unit ball in these deviations measures. For more detailed information and further references on best and random approximations of convex bodies by polytopes, we refer to the surveys by Bárány [1] and Schneider [13], and the books by Gruber [10] and Schneider [14]; the latter two also serve as references for fundamental properties of convex bodies.

\section{Proof of Theorem 1.2}

In this section we work in the hyperbolic plane $\mathbb{H}^{2}$, thus all notions, such as distance, convexity, perimeter, perimeter deviation, etc. are always understood in the hyperbolic sense without mentioning this fact explicitly. We think of $\mathbb{H}^{2}$ as a 2-dimensional Riemannian manifold of constant curvature -1 , such as the Beltrami-Klein model, see more on this below. By the curvature of a $C^{2}$ curve in $\mathbb{H}^{2}$ we mean its geodesic curvature. A compact set $K \subset \mathbb{H}^{2}$ is (geodesically) convex, if for any $x, y \in K$, the geodesic segment $x y$ is contained in $K$.

We follow an argument that is based on ideas of Eggleston in [5] but is somewhat more complicated due to the hyperbolic setting. First, note that the set of all compact, convex sets forms a complete metric space with respect to the Hausdorff distance in $\mathbb{H}^{2}$. Furthermore, the perimeter deviation function is continuous on this space. Thus, it is enough to prove the theorem on a suitable dense subspace of convex discs. We select this dense subspace the following way: We assume that the boundary bd $K$ of $K$ is $C_{+}^{2}$-smooth, meaning that it is twice continuously differentiable at every point and the geodesic curvature is strictly positive everywhere.

We start the proof by examining the difference between the length of a chord and the corresponding arc of bd $K$ cut off by the chord.

In the following argument, we will work in the Beltrami-Klein model $D$ of the hyperbolic plane $\mathbb{H}^{2}$ whose points are the interior points of the unit radius circular disc $B^{2}$ centred at the origin, and whose lines (geodesics) are the Euclidean open line segments with endpoints on the boundary $S^{1}$ of $B^{2}$. We define the distance of two points $p, q \in D$ as 


$$
d_{H}(p, q)=\frac{|\ln (a b p q)|}{2}
$$

where $a$ and $b$ are the intersection points of the line $p q$ with $S^{1}$ such that $a$ is on the side of $p$ and $b$ is on the side of $q$. The symbol (abpq) denotes the cross-ratio of the points $a, b, p, q$ in this order. It is well known that the Gaussian curvature of this model is constant -1 , with this particular metric. Now, if $p(x, y)$ is a point of $D$, where $x$ and $y$ are its Euclidean coordinates in a Cartesian coordinate system centred at the origin, then the first fundamental form of $D$ is

$$
\mathrm{d} s^{2}=\frac{\left(1-y^{2}\right) \mathrm{d} x^{2}+2 x y \mathrm{~d} x \mathrm{~d} y+\left(1-x^{2}\right) \mathrm{d} y^{2}}{\left(1-x^{2}-y^{2}\right)^{2}}
$$

see, for example, [3].

Let $K \subset D$ be a (geodesically) convex disc in $D$ whose boundary is $C_{+}^{2}$-smooth. Since geodesic segments in $D$ are exactly the Euclidean segments, the disc $K$ is convex in the hyperbolic sense exactly if it is convex in the Euclidean sense. Assume that $o \in$ bd $K$ and that the $x$-axis supports $K$ at $o$. Then, in a suitably small neighbourhood of $o$, the boundary of $K$ can be represented by a convex function $f$ such that $f(x)=$ $(\kappa / 2) x^{2}+o\left(x^{2}\right)$ as $x \rightarrow 0$, and $\kappa>0$. A standard calculation shows that the geodesic curvature of bd $K$ at $o$ is $\kappa$.

For sufficiently small $x$, let $s(x)$ denote the arc-length of bd $K$ between $o$ and the point $(x, f(x))$. Then

$$
\begin{aligned}
s(x) & =\int_{0}^{x} \frac{\left(1-f^{2}(\tau)+2 \tau f(\tau) f^{\prime}(\tau)+\left(1-\tau^{2}\right)\left(f^{\prime}(\tau)^{2}\right)\right)^{1 / 2}}{1-\tau^{2}-f^{2}(\tau)} \mathrm{d} \tau \\
& =\int_{0}^{x} \frac{\sqrt{1+\kappa^{2} \tau^{2}+o\left(\tau^{2}\right)}}{1-\tau^{2}+o\left(\tau^{2}\right)} \mathrm{d} \tau .
\end{aligned}
$$

After substituting the Taylor series of $\sqrt{1+z}$ around $z=0$ and that of $(1-z)^{-1}$ around $z=0$ in (1), we obtain

$$
\begin{aligned}
s(x) & =\int_{0}^{x}\left(1+\frac{\kappa^{2} \tau^{2}}{2}+o\left(\tau^{2}\right)\right)\left(1+\tau^{2}+o\left(\tau^{2}\right)\right) \mathrm{d} \tau \\
& =\int_{0}^{x}\left(1+\frac{\kappa^{2}+2}{2} \tau^{2}+o\left(\tau^{2}\right)\right) \mathrm{d} \tau=\left[\tau+\frac{\kappa^{2}+2}{6} \tau^{3}+o\left(\tau^{3}\right)\right]_{0}^{x} \\
& =x+\frac{\kappa^{2}+2}{6} x^{3}+o\left(x^{3}\right) \quad \text { as } x \rightarrow 0^{+}
\end{aligned}
$$

First, let $l=l(\delta)$ be the line with Euclidean equation $y=\delta$. For sufficiently small $\delta>0$, the line $l$ intersects bd $K$ at $x_{+}(\delta)>0\left(x_{-}(\delta)<0\right)$ such that $f\left(x_{+}(\delta)\right)=\delta$ $\left(f\left(x_{-}(\delta)\right)=\delta\right)$. Due to the definition of $f, x_{+}(\delta)=\delta^{1 / 2} \sqrt{2 / \kappa}+o\left(\delta^{1 / 2}\right)\left(x_{-}(\delta)=\right.$ $\left.-\delta^{1 / 2} \sqrt{2 / \kappa}+o\left(\delta^{1 / 2}\right)\right)$ as $\delta \rightarrow 0^{+}$. Thus, by (2), the arc of bd $K$ between $o$ and the positive intersection point of $l$ and bd $K$ has length 


$$
s\left(x_{+}(\delta)\right)=x_{+}(\delta)+\frac{\kappa^{2}+2}{6} x_{+}^{3}(\delta)+o\left(x_{+}^{3}(\delta)\right) \quad \text { as } \delta \rightarrow 0^{+} .
$$

Clearly, a similar formula holds for the length of the arc of bd $K$ between the intersection point with (negative) $x$-coordinate $x_{-}(\delta)$ and $o$.

The (hyperbolic) length of the segment between the $y$-axis and the (positive) intersection point with bd $K$ is

$$
\begin{aligned}
s_{l}(\delta) & =\int_{0}^{x_{+}(\delta)} \frac{\sqrt{1-\delta^{2}}}{1-x^{2}-\delta^{2}} \mathrm{~d} x=\frac{1}{2} \ln \frac{\sqrt{1-\delta^{2}}+x_{+}(\delta)}{\sqrt{1-\delta^{2}}-x_{+}(\delta)} \\
& =x_{+}(\delta)+\frac{x_{+}^{3}(\delta)}{3}+o\left(\delta^{2} x_{+}(\delta)\right) \quad \text { as } x_{+}(\delta) \rightarrow 0^{+},
\end{aligned}
$$

and, again, a similar formula holds for the length of the segment between the negative intersection point of $l$ and bd $K$ and the $y$-axis.

From (3) and (4), and the expressions for $x_{+}(\delta)$ and $x_{-}(\delta)$, we obtain that the difference of the arc of bd $K$ and the chord at (Euclidean) height $\delta$ is

$$
\frac{\kappa^{2}}{3}\left(x_{+}^{3}(\delta)+x_{-}^{3}(\delta)\right)+o\left(x_{+}^{3}(\delta)\right)+o\left(x_{-}^{3}(\delta)\right)=O\left(\delta^{3 / 2}\right) \quad \text { as } \delta \rightarrow 0^{+} .
$$

Since the hyperbolic height of $l$ is $\delta_{H}=\tanh ^{-1} \delta=\delta+O\left(\delta^{3}\right)$ as $\delta \rightarrow 0^{+}$, the conclusion of (4) holds with $\delta_{H}$ in place of $\delta$ as well.

Second, we assume that the Euclidean equation of the line $l$ is $y=\tan \theta \cdot x$, meaning that $l$ passes through $o$ and makes an angle $\theta$ with the positive part of the $x$-axis. If $\theta>0$ is sufficiently small, then for the $x$-coordinate $x(\theta)$ of the intersection point of $l$ and bd $K$, different from $o$, the following holds:

$$
f(x(\theta))=\tan \theta \cdot x(\theta)
$$

from which we obtain that

$$
x(\theta)=\frac{2 \tan \theta}{\kappa}+o(\tan \theta)=\frac{2 \theta}{\kappa}+o(\theta) \quad \text { as } \theta \rightarrow 0^{+} .
$$

Substituting $x(\theta)$ in (2), we get that the arc-length of bd $K$ between $o$ and the other intersection point of $l$ and bd $K$ is

$$
s(\theta)=x(\theta)+\frac{\kappa^{2}+2}{6} x^{3}(\theta)+o\left(x^{3}(\theta)\right) \quad \text { as } x(\theta) \rightarrow 0^{+} .
$$


At the same time, the length of the segment $l \cap K$ is

$$
\begin{aligned}
s_{l}(\theta) & =\tanh ^{-1} \sqrt{x^{2}(\theta)+f^{2}(x(\theta))} \\
& =\tanh ^{-1} \sqrt{x^{2}(\theta)+\tan ^{2} \theta \cdot x^{2}(\theta)}=\tanh ^{-1}(x(\theta) \sec \theta) \\
& =x(\theta) \sec \theta+\frac{x^{3}(\theta) \sec ^{3} \theta}{3}+O\left(x^{3}(\theta) \sec ^{3} \theta\right) \\
& =x(\theta)+\frac{x(\theta) \theta^{2}}{2}+\frac{x^{3}(\theta) \sec ^{3} \theta}{3}+O\left(x^{3}(\theta) \sec ^{3} \theta\right) .
\end{aligned}
$$

Now, by (6) and (7), the difference between the chord of $l$ and the corresponding part of bd $K$ is

$$
s(\theta)-s_{l}(\theta)=\left(\frac{8 \kappa^{2}}{6 \kappa^{3}}-\frac{1}{\kappa}\right) \theta^{3}+o\left(\theta^{3}\right)=\frac{\theta^{3}}{3 \kappa}+o\left(\theta^{3}\right)=O\left(\theta^{3}\right) \text { as } \theta \rightarrow 0^{+} .
$$

The observations (5) and (8) are elementary and known. We only included their detailed proofs because we could not find an explicit argument in the literature.

Now, we turn to the actual proof of Theorem 1.2. Let $P \in \mathcal{P}_{H}(n)$ be an $n$-gon which minimises the perimeter deviation from $K$, that is, $\operatorname{dev}(K, P)=\delta_{\operatorname{dev}_{H}}(K, n)$. We will denote the vertices of $P$ by $x_{1}, \ldots, x_{n}$ in a counter-clockwise cyclic order along $P$. It is clear that each side $x_{i} x_{i+1}$ has a common point with $K$, otherwise we could move it inwards and decrease the perimeter deviation using the monotonicity of perimeter in the hyperbolic plane, cf. [11, Prop. 1.3]. The assumption that bd $K$ is $C_{+}^{2}$ yields that $K$ is strictly convex, that is, bd $K$ contains no geodesic segment, and that bd $K$ has a unique supporting line at each point, and therefore it cannot have vertices.

The proof of Theorem 1.2 is indirect: we assume, on the contrary, that $P$ is not inscribed in $K$ and seek a contradiction. It is clear that if $P \subset K$, then the vertices of $P$ must be on bd $K$, similarly to the Euclidean case, for otherwise we could increase the perimeter of $P$ by moving the vertices out to the boundary of $K$. Therefore, the indirect assumption yields that $P$ has a side with at least one endpoint outside of $K$. There are several possibilities how this may happen. We treat each such case and show that they all contradict the best approximation property of $P$.

We use the following notation, similar to [5, Sect. 2]. Let the vertex $x_{i}$ be outside of $K$. We denote the internal angle of $P$ at $x_{i}$ by $\alpha_{i}$. Let $b_{i}$ be the last common point of the side $x_{i-1} x_{i}$ and bd $K$, and let $c_{i}$ be the first common point of $x_{i} x_{i+1}$ and bd $K$ in the counter-clockwise direction along $P$. Let the angle of the tangent of bd $K$ at $b_{i}$ and $x_{i-1} x_{i}$ be denoted by $\beta_{i}$, and the angle of the tangent of bd $K$ at $c_{i}$ and $x_{i} x_{i+1}$ be $\gamma_{i}$. Then, clearly, $\alpha_{i}+\beta_{i}+\gamma_{i}<\pi$.

Let us first consider the case when $P$ has a side, say $x_{1} x_{2}$, such that both $x_{1}$ and $x_{2}$ are outside of $K$. Let $\delta>0$ be small and let $h=h(\delta)$ be the hypercycle that is the equidistant curve from the line $x_{1} x_{2}$ at distance $\delta$ in the half-plane of $x_{1} x_{2}$ containing $P$. Let $x_{1}^{\prime}$ and $x_{2}^{\prime}$ be the intersection points of the sides $x_{n} x_{1}$ and $x_{2} x_{3}$ with $h(\delta)$, respectively. Assume that $\delta$ is so small that both $x_{1}^{\prime}$ and $x_{2}^{\prime}$ are outside of $K$. Then the $n$-gon $P^{\prime}$ with vertices $x_{1}^{\prime}, x_{2}^{\prime}, x_{3}, \ldots, x_{n}$, in this order, is contained in $P$, 
and the intersection of the side $x_{1}^{\prime} x_{2}^{\prime}$ and $K$ is of positive length. Let the feet of the perpendiculars from $x_{1}^{\prime}$ and $x_{2}^{\prime}$ to $x_{1} x_{2}$ be $x_{1}^{\prime \prime}$ and $x_{2}^{\prime \prime}$, respectively. Then $x_{1}^{\prime \prime} x_{2}^{\prime \prime} x_{2}^{\prime} x_{1}^{\prime}$ is a Saccheri quadrilateral. It is known that the line through the midpoints of the segments $x_{1}^{\prime} x_{2}^{\prime}$ and $x_{1}^{\prime \prime} x_{2}^{\prime \prime}$ is perpendicular to both lines, and thus it cuts $x_{1}^{\prime \prime} x_{2}^{\prime \prime} x_{2}^{\prime} x_{1}^{\prime}$ into two congruent Lambert quadrilaterals. Using known trigonometric relations for Lambert quadrilaterals, we obtain that

$$
\sinh \frac{d\left(x_{1}^{\prime \prime}, x_{2}^{\prime \prime}\right)}{2}=\sinh \frac{d\left(x_{1}^{\prime}, x_{2}^{\prime}\right)}{2} \cosh \delta,
$$

from which it follows that

$$
d\left(x_{1}^{\prime}, x_{2}^{\prime}\right)=d\left(x_{1}^{\prime \prime}, x_{2}^{\prime \prime}\right)+O\left(\delta^{2}\right) \quad \text { as } \delta \rightarrow 0^{+} .
$$

By hyperbolic trigonometry, we obtain for $i=1,2$ that

$$
\sinh d\left(x_{i}, x_{i}^{\prime}\right)=\sinh \delta \csc \alpha_{i},
$$

thus

$$
d\left(x_{i}, x_{i}^{\prime}\right)=\delta \csc \alpha_{i}+O\left(\delta^{3}\right) \quad \text { as } \delta \rightarrow 0^{+},
$$

and

$$
\sinh d\left(x_{i}, x_{i}^{\prime \prime}\right)=-\tanh \delta \cot \alpha_{i},
$$

thus

$$
d\left(x_{i}, x_{i}^{\prime \prime}\right)=-\delta \cot \alpha_{i}+O\left(\delta^{3}\right) \quad \text { as } \delta \rightarrow 0^{+} .
$$

If $x_{1} x_{2}$ is tangent to $K$ at a relative interior point $x^{\prime} \in x_{1} x_{2}$, then let $a$ and $b$ denote the intersection points of the segment $x_{1}^{\prime} x_{2}^{\prime}$ with bd $K$ such that $a$ is closer to $x_{1}^{\prime}$. Then $d\left(x^{\prime}, a b\right) \leq \delta$. By the positivity of the geodesic curvature of bd $K$ at $x^{\prime}$ and by (5), it holds that the difference of the arc-length of bd $K$ between $a$ and $b$ and the length of the segment $a b$ is $O\left(\delta^{3 / 2}\right)$ as $\delta \rightarrow 0$, and thus, by using the estimates obtained above, we get that

$$
\operatorname{dev}\left(K, P^{\prime}\right)=\operatorname{dev}(K, P)-\delta\left(\csc \alpha_{1}+\csc \alpha_{2}+\cot \alpha_{1}+\cot \alpha_{2}\right)+O\left(\delta^{3 / 2}\right)
$$

as $\delta \rightarrow 0^{+}$. Since $\csc \alpha+\cot \alpha \geq 0$ for any $\alpha \in(0, \pi)$, the coefficient of $\delta$ is negative in the above expression. This contradicts the minimality of $P$, and thus $P$ cannot have such a side.

If the side $x_{1} x_{2}$ cuts the boundary at two distinct points that are relatively interior to $x_{1} x_{2}$, then, according to the previously introduced notation, these intersection points are $c_{1}$ and $b_{2}$, and the tangents to bd $K$ make an angle $\gamma_{1}$ and $\beta_{2}$ with $x_{1} x_{2}$, respectively. We introduce the following notations, see Fig. 1. Let the last intersection point of $x_{1}^{\prime} x_{2}^{\prime}$ 


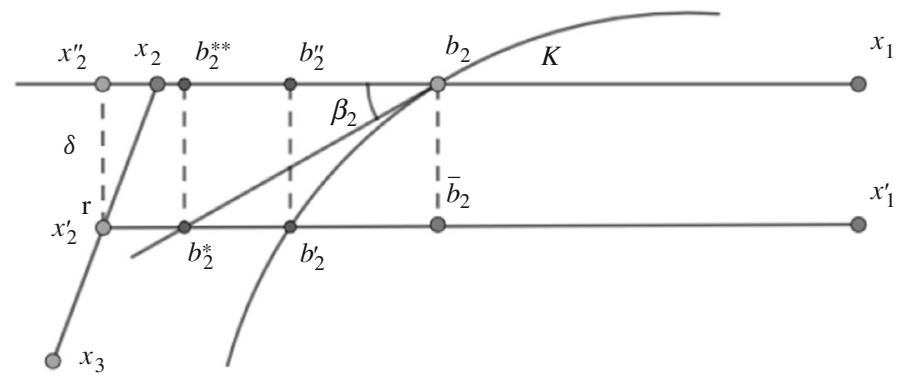

Fig. 1 The side $x_{1} x_{2}$ intersects the boundary of $K$ in two distinct interior points

and bd $K$ be $b_{2}^{\prime}$. Let $b_{2}^{\prime \prime}$ be the perpendicular projection of $b_{2}^{\prime}$ onto $x_{1} x_{2}$. Let $\bar{b}_{2}$ be the point on $x_{1}^{\prime} x_{2}^{\prime}$ whose perpendicular projection onto $x_{1} x_{2}$ is $b_{2}$. Let $b_{2}^{*}$ be the intersection point of the tangent line of bd $K$ through $b_{2}$ and $x_{1}^{\prime} x_{2}^{\prime}$. Finally, let $b_{2}^{* *}$ be the perpendicular projection of $b_{2}^{*}$ onto $x_{1} x_{2}$.

We first note that, using hyperbolic trigonometry, we can conclude that

$$
d\left(b_{2}, b_{2}^{\prime \prime}\right)=d\left(\bar{b}_{2}, b_{2}^{\prime}\right)+O\left(\delta^{2}\right) \quad \text { as } \delta \rightarrow 0^{+},
$$

and similarly,

$$
d\left(b_{2}^{\prime \prime}, b_{2}^{* *}\right)=d\left(b_{2}^{\prime}, b_{2}^{*}\right)+O\left(\delta^{2}\right) \quad \text { as } \delta \rightarrow 0^{+} .
$$

Now, let $\delta^{\prime}=d\left(b_{2}^{*}, b_{2}^{* *}\right)$. Clearly, $\delta^{\prime}<\delta$. Similarly as above, we obtain by hyperbolic trigonometry applied to the triangle $b_{2} b_{2}^{*} b_{2}^{* *}$ that

$$
\sinh d\left(b_{2}, b_{2}^{*}\right)=\sinh \delta^{\prime} \csc \beta_{2},
$$

from where

$$
d\left(b_{2}, b_{2}^{*}\right)=\delta^{\prime} \csc \beta_{2}+O\left(\delta^{\prime 3}\right) \quad \text { as } \delta \rightarrow 0^{+}
$$

moreover,

$$
\sinh d\left(b_{2}, b_{2}^{* *}\right)=-\tanh \delta^{\prime} \cot \beta_{2}
$$

and

$$
d\left(b_{2}, b_{2}^{* *}\right)=-\delta^{\prime} \cot \beta_{2}+O\left(\delta^{\prime 3}\right) \quad \text { as } \delta \rightarrow 0^{+} .
$$

From trigonometric formulae for the corresponding Lambert quadrilateral we get that

$$
\delta^{\prime}=\delta+O\left(\delta^{3}\right) \quad \text { as } \delta \rightarrow 0^{+} .
$$


Also, it is clear from the $C_{+}^{2}$ property of bd $K$ that

$$
d\left(b_{2}^{\prime}, b_{2}^{*}\right)=O\left(d\left(b_{2}, b_{2}^{*}\right)\right)=O\left(\delta^{2}\right) \quad \text { as } \delta \rightarrow 0^{+},
$$

and thus from all of the above,

$$
d\left(b_{2}, b_{2}^{\prime \prime}\right)=d\left(b_{2}, b_{2}^{* *}\right)+O\left(\delta^{2}\right) \text { and } d\left(\bar{b}_{2}, b_{2}^{\prime}\right)=d\left(\bar{b}_{2}, b_{2}^{*}\right)+O\left(\delta^{2}\right)
$$

as $\delta \rightarrow 0^{+}$. Let $l(\delta)$ denote the length of the arc of bd $K$ between $b_{2}$ and $b_{2}^{\prime}$. From (2) we obtain that

$$
l(\delta)-d\left(b_{2}, b_{2}^{*}\right)=O\left(\delta^{2}\right) \quad \text { as } \delta \rightarrow 0^{+} .
$$

Finally, putting everything together, we obtain (similarly as in $[5,(19)]$ ) that

$$
\begin{aligned}
\operatorname{dev}_{H}\left(K, P^{\prime}\right)= & \operatorname{dev}_{H}(K, P)-\delta\left(2 \cot \beta_{2}-2 \csc \beta_{2}+\csc \alpha_{2}+\cot \alpha_{2}\right. \\
& \left.+2 \cot \gamma_{1}-2 \csc \gamma_{1}+\csc \alpha_{1}+\cot \alpha_{1}\right)+O\left(\delta^{2}\right) \text { as } \delta \rightarrow 0^{+},
\end{aligned}
$$

and thus, by the optimality of $P$, it must hold that

$$
\cot \frac{\alpha_{1}}{2}+\cot \frac{\alpha_{2}}{2}=2\left(\tan \frac{\beta_{2}}{2}+\tan \frac{\gamma_{1}}{2}\right) .
$$

In the following case we do not give all details of the calculations as those are very similar to the ones discussed above. We rather just point out the main conclusions of these calculations.

Next, assume that for the side $x_{1} x_{2}$ it holds that $x_{1} \in K$ and $x_{2} \notin K$. Rotate the line $x_{1} x_{2}$ around $x_{1}$ by a sufficiently small positive angle $\varphi$ so that the intersection point $x_{2}^{\prime}$ of the rotated line with the side $x_{2} x_{3}$ is still outside $K$. Let $P^{\prime}$ be the polygon with vertices $x_{1}, x_{2}^{\prime}, x_{3}, \ldots, x_{n}$. Clearly, $P^{\prime} \subset P$. Let $b_{2}$ be the last intersection point of the side $x_{1} x_{2}$ with bd $K$, as before.

If the line $x_{1} x_{2}$ is not a supporting line of $K$ at $x_{1}$, then we obtain by hyperbolic trigonometry that

$$
\begin{aligned}
\operatorname{dev}\left(K, P^{\prime}\right)= & \varphi\left(2\left(\csc \beta_{2}-\cot \beta_{2}\right) \sinh d\left(x_{1}, b_{2}\right)-\left(\csc \alpha_{2}+\cot \alpha_{2}\right) \sinh d\left(x_{1}, x_{2}\right)\right) \\
& +\operatorname{dev}(K, P)+O\left(\varphi^{2}\right) \quad \text { as } \varphi \rightarrow 0^{+} .
\end{aligned}
$$

Due to the optimality of $P$, it must hold that

$$
\frac{\sinh d\left(x_{1}, x_{2}\right)}{\sinh d\left(x_{1}, b_{2}\right)} \cot \frac{\alpha_{2}}{2}=2 \tan \frac{\beta_{2}}{2} .
$$

Note that $d\left(x_{1}, x_{2}\right)>d\left(x_{1}, b_{2}\right)$, and thus by the strictly monotonically increasing property of the sinh function it follows that the coefficient of $\cot \left(\alpha_{2} / 2\right)$ in (10) is larger than 1 . 
If $x_{1} x_{2}$ is a supporting line of $K$, then, using (8), we get that

$$
\operatorname{dev}\left(K, P^{\prime}\right)=\operatorname{dev}(K, P)-\varphi\left(\csc \alpha_{2}+\cot \alpha_{2}\right) \sinh d\left(x_{1}, x_{2}\right)+O\left(\varphi^{2}\right)
$$

as $\varphi \rightarrow 0^{+}$. As the coefficient of $\varphi$ is negative, this clearly contradicts the minimality of $P$, so $P$ cannot have such a side.

Now, the proof can be finished as in [5, p.357, (24)-(25)]: For each $x_{i} \notin K$, the angle $\alpha_{i}$ appears in exactly two equations of type (9) or (10), and $\beta_{i}$ and $\gamma_{i}$ in exactly one such equation. Thus, by adding the two equations in which $\alpha_{i}$ appears, the coefficient of $\cot \left(\alpha_{i} / 2\right)$ will be at least 2 . If $2+\varepsilon_{i}$ denotes the coefficient of $\cot \left(\alpha_{i} / 2\right)$, then summing all equations of type (9) and (10) yields that

$$
\begin{aligned}
\sum\left(2+\varepsilon_{i}\right) \cot \frac{\alpha_{i}}{2} & =\sum 2\left(\tan \frac{\beta_{i}}{2}+\tan \frac{\gamma_{i}}{2}\right)<\sum 2 \tan \frac{\beta_{i}+\gamma_{i}}{2} \\
& \leq \sum 2 \tan \frac{\pi-\alpha_{i}}{2}=\sum 2 \cot \frac{\alpha_{i}}{2}
\end{aligned}
$$

which is clearly a contradiction as all $\varepsilon_{i} \geq 0$. This finishes the proof of Theorem 1.2.

\section{Counterexample on the Sphere}

It is known that among spherical triangles contained in a (spherical) circle the inscribed regular triangle has the maximal perimeter, cf. László Fejes Tóth [6]. Thus, among triangles contained in the circle, the inscribed regular one has the minimum perimeter deviation from the circle. However, below we show an example of a triangle and circle, where the triangle is neither inscribed nor circumscribed, and approximates the circle better than either the inscribed or the circumscribed regular triangle.

Let $K(r)$ be the spherical circle with centre $P$ and radius $r$. Consider the regular spherical triangle $T(d)=A B C \triangle$ with centre $P$ and circumradius $d$. Let $l=l(d)$ denote the side length of $T(d)$ and $m=m(d)$ its inradius, see Fig. 2. Then

$$
\cos l=\cos ^{2} d+\sin ^{2} d \cos \frac{2 \pi}{3} \quad \text { and } \quad \cos m=\frac{\cos d}{\cos (l / 2)} .
$$

Let $D$ be the intersection point of the side $B C$ with the circle $K(r)$ that is closer to $B$. Let $E$ be the intersection of the side $B C$ and the great circle through $P$ perpendicular to $B C$. Then $m$ is the distance between $P$ and $E$. Denote by $s=s(d)$ the length of the arc between $D$ and $E$. Furthermore, let $\alpha=\alpha(d)$ be the central angle $\angle E P D$. Then

$$
\cos s=\frac{\cos r}{\cos m} \quad \text { and } \quad \cos \alpha=\frac{\cos s-\cos m \cos r}{\sin m \sin r} .
$$

Thus

$$
f(r, d)=\operatorname{dev}_{S}(K(r), T(d))=6\left(2 \alpha(d) \sin r-2 s(d)+\frac{l(d)}{2}\right)-2 \pi \sin r .
$$


Fig. 2 The spherical triangle $A B C \triangle$

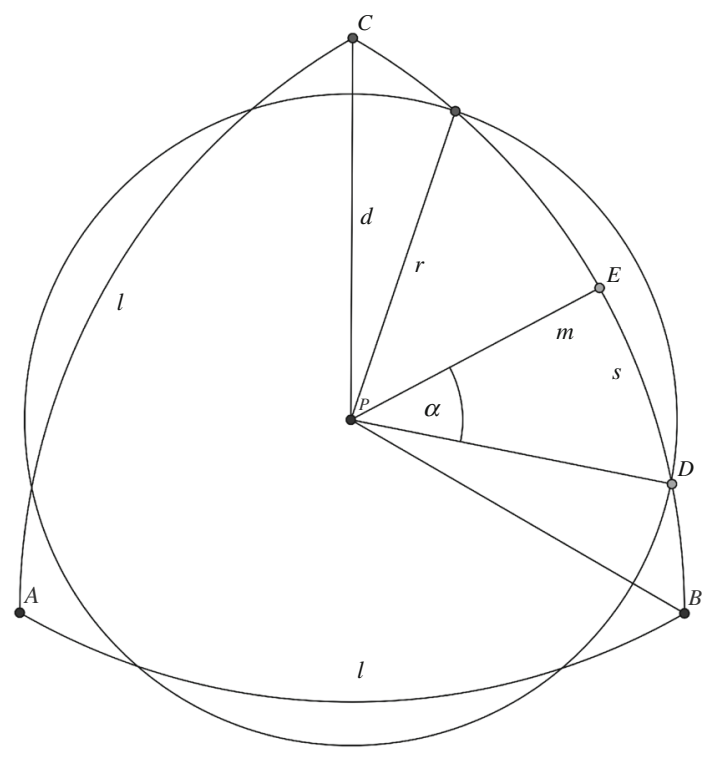

Fig. 3 The graph of $f(\pi / 2-0.1, d)$ over the interval $[\pi / 2-0.1,1.52]$

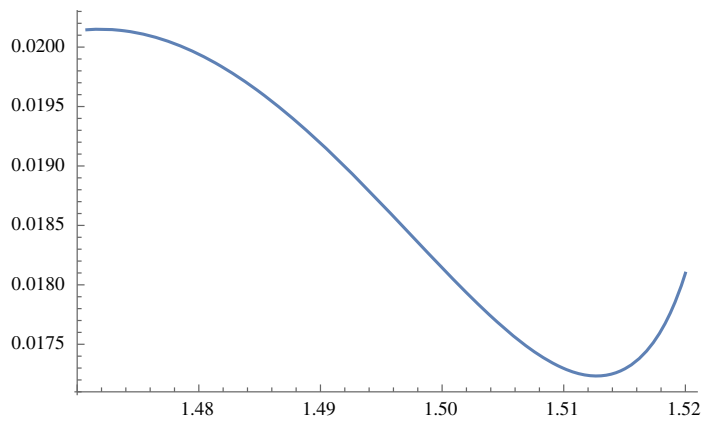

The graph of $f(\pi / 2-0.1, d)$ over the interval $[\pi / 2-0.1,1.52]$ is shown in Fig. 3, which clearly has its minimum inside the interval. We note that at the left endpoint of the interval the triangle is inscribed and at the right endpoint it is circumscribed. In fact, the circumscribed regular triangle approximates $K(r)$ better than the inscribed one, and the minimum occurs for a triangle that is neither inscribed nor circumscribed. Since all of these triangles are contained in the open hemisphere centred at $P$, they are convex in the spherical sense.

Acknowledgements This research was supported by grant TUDFO/47138-1/2019-ITM of the Ministry for Innovation and Technology, Hungary, and by Hungarian National Research, Development and Innovation Office NKFIH grant K116451. The author is grateful to Professor Gábor Fejes Tóth (Budapest, Hungary) for the enlightening discussions. The author also wishes to thank the MTA Alfréd Rényi Mathematical Research Institute where part of this research was done.

Funding Open access funding provided by the University of Szeged Open Access Fund grant no. 5230. 
Open Access This article is licensed under a Creative Commons Attribution 4.0 International License, which permits use, sharing, adaptation, distribution and reproduction in any medium or format, as long as you give appropriate credit to the original author(s) and the source, provide a link to the Creative Commons licence, and indicate if changes were made. The images or other third party material in this article are included in the article's Creative Commons licence, unless indicated otherwise in a credit line to the material. If material is not included in the article's Creative Commons licence and your intended use is not permitted by statutory regulation or exceeds the permitted use, you will need to obtain permission directly from the copyright holder. To view a copy of this licence, visit http://creativecommons.org/licenses/by/4.0/.

\section{References}

1. Bárány, I.: Random polytopes, convex bodies, and approximation. In: Stochastic Geometry. Lecture Notes in Mathematics, vol. 1892, pp. 77-118. Springer, Berlin (2007)

2. Besau, F., Hoehner, S., Kur, G.: Intrinsic and dual volume deviations of convex bodies and polytopes. Int. Math. Res. Not. (2019). https://doi.org/10.1093/imrn/rnz277

3. Cannon, J.W., Floyd, W.J., Kenyon, R., Parry, W.R.: Hyperbolic geometry. In: Flavors of Geometry. Mathematical Sciences Research Institute Publications, vol. 31, pp. 59-115. Cambridge University Press, Cambridge (1997)

4. Dowker, C.H.: On minimum circumscribed polygons. Bull. Am. Math. Soc. 50, 120-122 (1944)

5. Eggleston, H.G.: Approximation to plane convex curves. I. Dowker-type theorems. Proc. Lond. Math. Soc. 7, 351-377 (1957)

6. Fejes Tóth, L.: Lagerungen in der Ebene auf der Kugel und im Raum. Grundlehren der Mathematischen Wissenschaften, vol. 65. Springer, Berlin (1953)

7. Fejes Tóth, L.: Remarks on polygon theorems of Dowker. Mat. Lapok 6, 176-179 (1955). (in Hungarian)

8. Fejes Tóth, L.: Annäherung von Eibereichen durch Polygone. Math.-Phys. Semesterber. 6, 253-261 (1958/1959)

9. Florian, A.: The perimeter deviation of a convex set from a polygon. Rend. Circ. Mat. Palermo Suppl. 80, 169-181 (2008)

10. Gruber, P.M.: Convex and Discrete Geometry. Grundlehren der Mathematischen Wissenschaften, vol. 336. Springer, Berlin (2007)

11. Klain, D.A.: Bonnesen-type inequalities for surfaces of constant curvature. Adv. Appl. Math. 39(2), 143-154 (2007)

12. Molnár, J.: On inscribed and circumscribed polygons of convex regions. Mat. Lapok 6, 210-218 (1955). (in Hungarian)

13. Schneider, R.: Discrete aspects of stochastic geometry. In: Handbook of Discrete and Computational Geometry. CRC Press Ser. Discrete Math. Appl., pp. 167-184. CRC, Boca Raton (1997)

14. Schneider, R.: Convex Bodies: the Brunn-Minkowski Theory. Encyclopedia of Mathematics and Its Applications, vol. 151. Cambridge University Press, Cambridge (2014)

Publisher's Note Springer Nature remains neutral with regard to jurisdictional claims in published maps and institutional affiliations. 